\title{
Recortes espaciales que configuran el Oriente antioqueño: de la región a la superposición de territorialidades ${ }^{*}$
}

\section{Spatial Clipping Configurating East Antioquia: from Regional to Overlapping of Territorialities}

Recortes espaciais que compõem o Leste da Antioquia: da região à sobreposiçãa de territorialidades

Hernán Darío Pineda Gómez** Alejandro Pimienta Betancur ${ }^{\star \star \star}$

Recibido: 23 de noviembre de 2020

Aprobado: 25 de marzo de 2021

https://doi.org/10.12804/revistas.urosario.edu.co/territorios/a.9946

Para citar este artículo

Pineda Gómez, H. D., \& Pimienta Betancur, A. (2021). Recortes espaciales que configuran el Oriente antioqueño: de la región a la superposición de territorialidades. Territorios (45), pp. 41-62. https://doi. org/10.12804/revistas.urosario.edu.co/territorios/a.9946

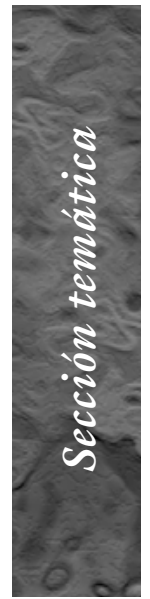

* Este articulo es un avance de la tesis doctoral Territorialidades de la globalización: localización industrial y reconfiguraciones en el Valle de San Nicolás, Colombia, desarrollada por Hernán Darío Pineda, dirigida por el Dr. Alejandro Pimienta Betancur, coautor de este artículo. La investigación se desarrolla en el doctorado en Ciencias Sociales de la Universidad de Antioquia, desde el Grupo de Estudios del Territorio del Instituto de Estudios Regionales INER.

** Docente ocasional del Instituto de Estudios Regionales-INER. Investigador del Grupo Estudios del Territorio (UdeA). Correo electrónico: hernan.pineda@udea.edu.co, ORCID: https://orcid.org/00000002-5691-7390 
Palabras clave

Movimiento industrial; oriente antioqueño; superposición de

territorialidades; efectos territoriales; recorte territorial.

Keywords

Industrial movement; eastern Antioquia; superposition of territorialities; territorial effects; territorial cut.

Palavras-chave Movimento industrial; Leste da Antioquia; sobreposição de territorialidades; efeitos territoriais; recorte territorial.

\section{territarias 45}

\section{RESUMEN}

El proceso de transformación del Oriente antioqueño y su configuración como espacio de la globalización permite entenderlo hoy como una suma de recortes espaciales cruzados por una superposición de territorialidades, que tensionan el uso y apropiación de dicho espacio en función de los múltiples intereses de regirlo, ordenarlo y envolverlo. El detonante de este proceso está anclado a un proyecto de regionalización funcional derivado del movimiento industrial desde la década de los 70, el cual fue forjando polígonos de borde, fragmentos subsidiarios de un proyecto de competitividad-desarrollista que se mantiene actualmente. De forma paralela, en diferentes momentos históricos han ido emergiendo territorialidades de diversa índole: unas solidarias con el proyecto económico, asociadas a una idea de intervención en diversas escalas; y otras, de la contigüidad, ligadas a un proyecto político social que reivindica la autonomía de la metrópoli, con una apuesta más horizontal. Por ello la idea de Oriente como una región administrativa homogénea, que aún es usada en procesos de planeación, carece de sustento geográfico y político.

\section{ABSTRACT}

The transformation process of Eastern Antioquia and its configuration as a space of globalization allows us to understand it today as a sum of spatial cuts crossed by a superposition of territorialities, which stress the use and appropriation of this space according to the multiple interests of governing, ordering and enveloping it. The trigger of this process is anchor to a project of functional regionalization derived from the industrial movement since the $70 \mathrm{~s}$, which was forging edge polygons, subsidiary fragments of a project of competitiveness development that is maintained today. In parallel, at different historical moments, territorialities of different kinds have been emerging: some in solidarity with the economic project, associated with an idea of intervention at different scales; and others, from contiguity, linked to a social-political project that claims autonomy from the metropolis, with a more horizontal bet. Therefore, the idea of the Oriente as a homogeneous administrative region, which is still used in planning processes, lacks geographical and political support.

\section{RESUMO}

O processo de transformação do Leste da Antioquia e sua configuração como espaço de globalização permite compreendê-lo hoje como um somatório de recortes espaciais atravessados por uma sobreposição de territorialidades, que acentuam o uso e a apropriação desse espaço a partir dos múltiplos interesses de governá-lo, ordená-lo e envolvê-lo. O gatilho desse processo está ancorado em um projeto de regionalização funcional derivado do movimento industrial desde a década de 1970, o qual foi forjando polígonos de ponta, fragmentos subsidiários de um projeto de competitividade-desenvolvimentista que se mantém até hoje. Paralelamente, em diferentes momentos históricos, emergiram diferentes tipos de territorialidades: algumas solidárias com o projeto econômico, associadas à uma ideia de intervenção em várias escalas; e outras, de contiguidade, vinculadas a um projeto político-social que reivindica a autonomia 
da metrópole, com uma aposta mais horizontal. Por isso, a ideia do Leste da Antioquia como uma região administrativa homogênea, que ainda é utilizada em processos de planejamento, carece de respaldo geográfico e político.

\section{Presentación}

El presente artículo plantea la tesis que en el Oriente antioqueño se ha producido una territorialidad hegemónica que ha forjado un espacio de la globalización - en los términos planteados por Santos (1993) y Silveira (2008) — vinculada al proyecto económico. Sin embargo, persisten otras lógicas territoriales que se han ido moldeando por este fenómeno, lo que permite interpretar su configuración actual como una sumatoria de recortes espaciales que no corresponden a una sola regionalización.

Para sustentar esta premisa, en primer lugar, se aborda la perspectiva teóricometodológica fundada principalmente en la geografía crítica y los estudios socioespaciales. En segundo lugar, se explica la relación geohistórica entre el Valle de San Nicolás y el Valle de Aburrá, y el movimiento industrial hacia esta subregión administrativa, sus formas de ocupación y las apuestas de intervención normativas. Finalmente, se presentan los efectos de este proceso en la configuración actual del Valle de San Nicolás.

\section{Perspectiva teórica}

Desde la perspectiva de la geografía crítica y los estudios socioespaciales, la reconfiguración territorial a partir de procesos promovidos globalmente, como la relocalización industrial y la respectiva intervención de espacios locales, implica una combinación de formas de producir el territorio, que, a su vez, genera cambios para dinámicas preexistentes. En este sentido, la presencia de flujos globales y sus anclajes espaciales no se asume como una destrucción de lo local, por el contrario, emerge allí un espacio de territorialidades, donde la agregación de nuevos elementos artificiales y la acción política que adapta los existentes dan origen a un paisaje particular, a una nueva forma ligada a una funcionalidad diferente, siendo un proceso dinámico, en movimiento.

Lo anterior permite ubicar un posicionamiento teórico/metodológico para examinar el fenómeno abordado. En un primer momento, se considera la producción de espacios de la globalización como detonante de los cambios, lo que posibilita interpretar la particularidad del proceso en el Oriente antioqueño y la manera en que el proyecto político ligado al movimiento industrial envuelve la subregión. En un segundo momento, se aborda la reflexión sobre los procesos de regionalización y la producción de diferenciación geográfica, lo que implica una forma de interpretar los recortes territoriales producidos por las diversas lógicas nales-INER-Investigador del Grupo Estudios del Territorio (UdeA). Correo electrónico: alejandro.pimienta@udea.edu. co, ORCID: https://orcid. org/0000-0002-74828965 
territoriales presentes en esta subregión. Para ello, se aborda la discusión sobre la región y la regionalización, lo que permite tensionar la idea de Oriente como un proceso homogéneo y plantear la tesis de la superposición de territorialidades donde se expresan múltiples ideas de región.

\section{Los espacios de la globalización y el uso del territorio}

De acuerdo con las discusiones desarrolladas por Santos (1993) y Silveira (2007; 2008), la globalización continúa siendo un paradigma para la comprensión de la realidad, resaltando como característica del periodo actual la imposición de una forma de producir, cuyo elemento central es la disociación de los procesos productivos y su unificación como un producto global. Nuevas centralidades, nuevas periferias, nuevas formas de desconcentración productiva son una expresión de esta dinámica que desborda las grandes aglomeraciones e irradia los espacios adyacentes. Este fenómeno no opera en todos los lugares ni de igual forma, ni de manera simultánea, por tal motivo se hace necesario hacer énfasis en comprender las particularidades que se producen en cada lugar.

Lo anterior implica reconocer que la globalización organiza de una manera específica los fragmentos del espacio en función de los intereses de los actores hegemónicos (Santos \& Silveira, 2008), forjando los espacios de la globalización
(Santos, 1993). Estos se conciben como una organización intencional de recortes particulares en función de una productividad espacial, los cuales presentan cargas diferenciales de contenido y densidad técnica, enlazados a la dinámica global (Silveira, 2007). En este sentido, estos polígonos/recortes no son iguales a la división político-administrativa (municipios/ciudades), son fragmentos a su interior, articulados a una dinámica más amplia que puede tener diversos alcances (Silveira, 2014). Un punto de interés en esta discusión es la centralización de la articulación global y técnica, es decir, los recortes territoriales producidos como parte de la expansión de aglomeraciones urbanas pueden operar bajo una modalidad de centralización/multinodal, ya sea a partir de una serie de flujos transfronterizos (Sassen, 2007) o bajo una conexión local/regional y de allí, un salto a lo global. Esto configura una ruta para examinar los nuevos procesos de desconcentración productiva, en este caso, una mirada a la relación entre Medellín y el Oriente antioqueño.

Aquí emerge otro punto de interés, no todas las acciones en el territorio son siempre iguales. Las empresas construyen su base material con diferentes principios de competitividad (Silveira, 2007), una idea de uso diferenciado del territorio, desplegando un movimiento desde/hacia el polígono industrial, ya sea a través de la demanda de materia prima, del movimiento de las mercancías y trabajadores, del 
flujo de órdenes para el direccionamiento productivo y del rol que desempeñan en una arquitectura productiva global. Esto produce una idea de enrejado territorial, proceso que no es homogéneo y que genera una superposición de intencionalidades y jerarquías (Silveira, 2007), un circuito diferenciado que envuelve los espacios locales. Lo anterior permite comprender que los movimientos sobre el territorio se pueden modificar, adaptar y transformar a partir de las topologías que cada empresa implementa (Silveira, 2008; 2014; Santos \& Silveira, 2008) —esto es, la relación entre los puntos de interés para su operación, lo que afecta a otras empresas y la imagen del lugar, ya que se empiezan a organizar de acuerdo con la lógica dominante-y las formas de darle funcionalidad a fragmentos, en este sentido se destaca el rol de la norma para impulsar y proscribir usos (Silveira, 2014).

En este punto se destaca que los ciclos de la globalización no son estáticos y lo que en un momento significó una ventaja comparativa, luego ya no lo es. Esto conlleva a procesos de valorización y desvaloración territorial ante las fluctuaciones globales, que producen diversos grados de inestabilidad en las variables locales (Silveira, 2007). Esta mirada sirve para comprender cómo las dinámicas territoriales quedan atrapadas a decisiones que se toman en lugares muy distantes (Silveira, 2014).

Finalmente, es importante resaltar que la forma como los espacios de la globalización recortan el territorio no implica que desaparezcan otras producciones territoriales. La territorialización a partir de fenómenos promovidos globalmente fragmenta el territorio de acuerdo con la topología y funcionalidad que se quiera imponer, pero allí siguen persistiendo formas de articulación zonal hacia adentro (Haesbaert, 2014), emergiendo una tensión por el uso del territorio. Ambas formas coexisten de forma superpuesta, aunque una de ellas hegemoniza la dinámica territorial. De esto resulta el interés por comprender los procesos de regionalización y superposición, como se observa a continuación.

\section{La articulación regional y las formas de envolver el lugar}

La discusión sobre formas de recorte territorial implica poner en tensión la idea de región como un proceso homogéneo, administrativo, estático, de contigüidad, y enfatizar en las diferentes formas de regionalización que se configuran (normativa, funcional, social). Desde este enfoque emerge una mirada de la región como una suma de retazos espaciales bajo una misma idea nominal, que para el objeto de estudio sería el Oriente antioqueño. En este sentido, se parte por un posicionamiento que ubica la región como un arte-facto (Haesbaert, 2019), a partir de las múltiples formas de la diferenciación geográfica (los procesos de 
Figura 1. La región como un Arte-facto

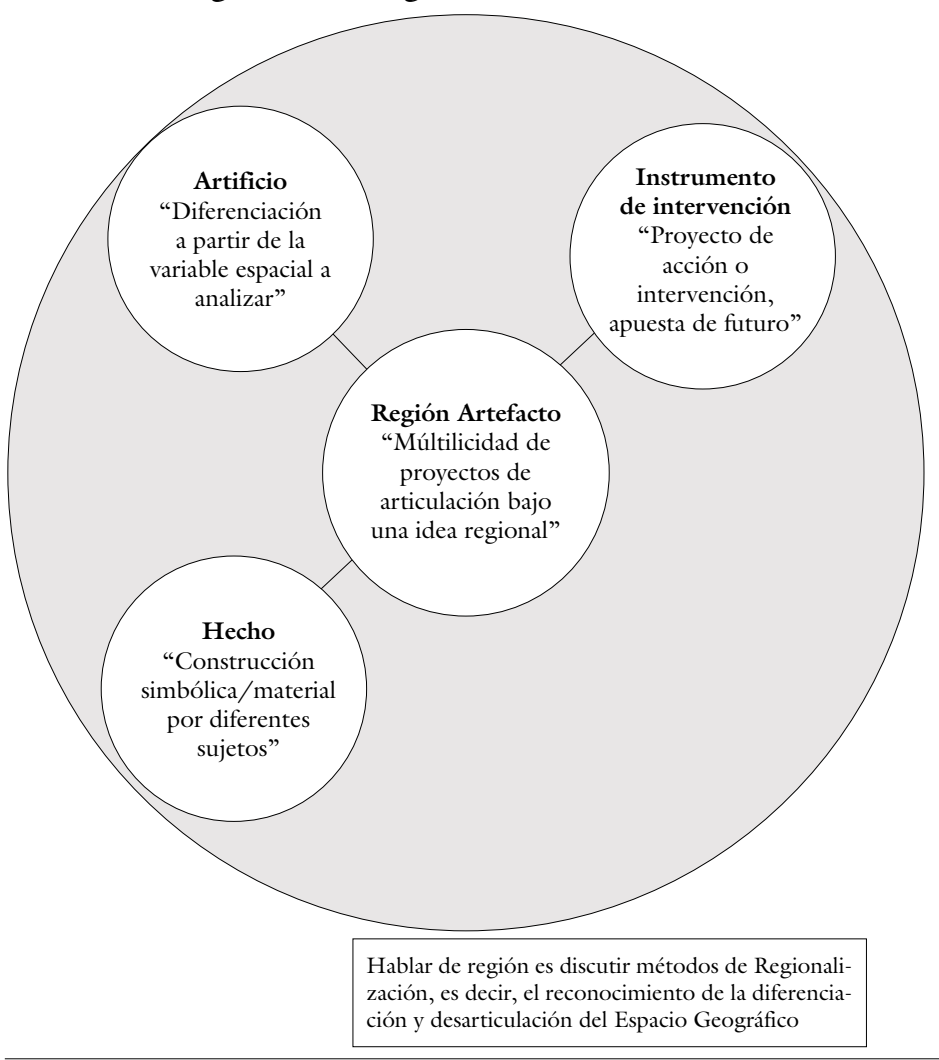

Fuente: elaboración propia a partir de Haesbaert $(2010 ; 2019)$.

\section{tersitarias 45}

práctica o simbólica, que es tanto un hecho, ideal-simbólico o material-funcional, como también una categoría de análisis de la diferenciación socioespacial para los investigadores. La figura 1 detalla la propuesta de Haesbaert y su valor analítico para comprender múltiples proyectos de articulación regional: a nivel simbólico, funcional, normativo o como artificio del investigador.

En esta vía, la región no es algo dado ni natural, sino que se configura como una producción social a partir de lógicas espaciales superpuestas, las cuales pueden ser zonales, reticulares o aglomerados (una idea de i-lógica espacial) (Haesbaert, 2019). Cualquier análisis regional debe tener en cuenta: la producción material, las representaciones y símbolos, la funcionalidad (político-económica) y su desdoble sobre una base material. Por tanto, la idea de región está relacionada con un contenido, unos actores y sus intencionalidades (Haesbaert, 2019).

En este sentido, Haesbaert (2010; 2019) plantea que la región se puede abordar desde la comprensión de los procesos de des-articulación del espacio, esto es, la forma en que los distintos proyectos de regionalización han producido recortes en el espacio geográfico. Lo anterior implica que, la regionalización, entendida como un "proceso analítico de reconocimiento de la diferenciación del espacio" (Haesbaert, 2010, p. 2), se configura como una ruta para comprender la multiplicidad de proyectos regionales, pero a su vez, 
para interpretar los procesos de territorialización, entendidos como proceso de apropiación concreta o simbólica del espacio (Haesbaert, 2014; 2019). Es decir, reconocer las formas de des-articulación del espacio implica analizar en función de qué relación de poder se está moldeando el mismo. En este sentido, los procesos de regionalización son una vía para comprender los procesos de diferenciación socioespacial, a su vez, cuando este análisis enfatiza en las prácticas de poder, supone una mirada sobre la territorialización (Haesbaert, 2019).

La anterior ruta no implica una tensión entre territorio y región, por el contrario, se interpretan como una perspectiva asociativa en la cual un concepto está al interior del otro. El reconocimiento de cada uno radica en el foco de lo que se está analizando, es decir, si la mirada está en las prácticas y el poder, hablamos de territorio, si es en los procesos de articulación o diferenciación socio-espacial, estamos mirando la región (Haesbaert, 2019).

De esta forma, la regionalización puede ser una mirada para comprender los procesos de diferenciación geográfica, lo cual permite poner de manifiesto intencionalidades, apuestas, proyectos diferenciados, lo que implica una producción geopolítica del territorio. A continuación, se analizan los proyectos superpuestos que configuran el Oriente antioqueño, los cuales son de tipo administrativo, funcional y social.

\section{Configuración geohistórica de la relación entre el Valle de Aburrá y el Valle de San Nicolás}

El Oriente antioqueño es una de las nueve subregiones administrativas del departamento de Antioquia, que fueron establecidas mediante Ordenanza 41 de 1975 de la Asamblea Departamental. Está subdividida en cuatro subzonas no homogéneas: páramos, bosques, altiplano (o Valle de San Nicolás) y embalses. Durante los 45 años de existencia de esta regionalización normativa, se han ido desplegando procesos institucionales que han pretendido ordenarla y regirla, así como una práctica social que ha incorporado esta noción de región en sus sentidos y significados territoriales, lo que ha conllevado a la formación de una idea de Oriente a nivel normativo, social y funcional (proyectos económicos).

Esta subregión integra 23 municipios y cuenta con 595.030 habitantes, de los cuales el $58,72 \%(349.425)$ corresponde a población urbana y el 41,28\% (245.605) a población rural, según datos del DANE del 2018. Según la distribución de la población en las cuatro zonas, el Valle de San Nicolás concentra la mayoría de ella con un $70,32 \%$ (418.402 habitantes), presentando los mayores niveles de urbanización (Montoya \& Carmona, 2020). Además, aporta el $8,53 \%$ al PIB departamental, siendo la segunda subregión administrativa con mayor dinámica 
empresarial y económica, después del área metropolitana del Valle de Aburrá, posición que ha conservado desde los años 80 (Villegas, 1988). De acuerdo con la Corporación Autónoma Regional de las Cuencas de los Ríos Negro y Nare, CORNARE (2016), "cerca del 76\% del valor agregado del Oriente antioqueño se concentra en el Valle de San Nicolás, en el corredor industrial de los municipios de El Retiro, El Santuario, Guarne, La Ceja, Marinilla, y Rionegro" (p. 24). Esto muestra que el proceso económico no irradia al conjunto de la subregión, sino que se ha concentrado en fragmentos.

La estructura y contenido actual del Valle de San Nicolás responde a una configuración geohistórica en relación con la estructuración de Medellín como núcleo del poder político y económico, y como centro de gravedad para todo el espacio que hoy comprende el departamento de Antioquia. Este proceso se remonta al siglo XVIII y XIX, cuando Medellín pasó de ser sitio de enlace comercial a capitalizar los excedentes de la minería del oro y el café, iniciando un proceso de industrialización (CENIC, 1988) y configurándose como capital del departamento, lo que conllevó a una combinación de la centralidad económica, burocrática y, a lo largo del siglo Xx, demográfica. De esta forma, en Medellín se inicia un proceso de cambio, al pasar de ser un nodo secundario, incluso periférico durante siglo XVIII, para convertirse en una ciudad en proceso de modernización a comienzos del siglo XX (Betancur et al., 2001), estructurándose como el epicentro de las apuestas y visiones de desarrollo a nivel departamental. Este nuevo centro de gravedad y sus demandas de bienes como agua, alimentos y energía eléctrica, así como las proyecciones de articulación a los mercados, envolvieron con diversa intensidad a las diferentes subregiones del departamento, sobre todo aquellas mejor conectadas con la capital.

Ejemplo de lo anterior es la articulación que se estructuró con la subregión del Oriente antioqueño. Los inicios de esta dinámica relacional se remontan a la colonización Antioqueña a finales del siglo XVIII, cuando el municipio de Sonsón (en la zona de páramos) fue el punto de conexión de Medellín hacia el viejo Caldas (Uribe, 1988); en el siglo XIX, fue punto de articulación hacia el centro del país por el Magdalena Medio, lo que propició una dinámica comercial e industrial en este municipio y en Rionegro, que ya era epicentro comercial para la época (CENIC, 1988). Allí empezaron a surgir industrias poco tecnificadas: en Rionegro, se desarrolló la industria asociada al cuero y al calzado; en Guarne, a la lana y la cabuya; en el Carmen de Viboral, a la cerámica; y en el Retiro, a los muebles. En todos estos casos se desarrollaron principalmente pequeñas y medianas empresas (Codesarrollo, 1976).

La conexión Medellín-Valle de San Nicolás, como parte de la integración de la capital con el río Magdalena, alcanzó 
a incluir el proyecto de movilidad del Tranvía de Oriente (figura 2), que inició la construcción en 1898 (CORNARE e INER, 1993b). Este proyecto tomó un nuevo aire en 1923 con la empresa Tranvía del Oriente, la cual tuvo como socios a Medellín, La Ceja, Marinilla, Cocorná, El Peñol, Granada, El Carmen, Guarne, San Vicente, San Carlos, Guatapé y El Santuario, pero solo se construyeron 51 de $300 \mathrm{~km}$ proyectados, suspendiéndose el proyecto en 1929 (CENIC, 1988). Si bien la articulación inicial al centro del país no se logró, sí fortaleció los vínculos y conexiones entre los dos Valles.

La dinámica comercial e industrial entre Medellín y el Oriente antioqueño se fue debilitando con la llegada del siglo XX (Uribe, 1988). La apuesta por la industrialización de Antioquia, centralizada en Medellín, así como el inicio de operaciones en 1914 del ferrocarril de Antioquia, fue moviendo el eje de conexión hacia el centro del país por el municipio de Puerto Berrio, subregión del Magdalena Medio. Esto desestructuró la conexión Medellín-Rionegro y, de paso, produjo una marginalización espacial de Sonsón, que todavía se mantiene. Medellín no solo se configuró como epicentro industrial y comercial del departamento, también fue foco de atracción para la fuerza de trabajo y los capitales que se habían forjado en la industria artesanal en el Oriente Antiqueño (IDOM, 2018). Incluso, nuevas industrias en la capital eran propiedad de empresarios de Rionegro (Uribe, 1988; Villegas, 1988).

Si bien, el proceso de vínculo entre los Valles proviene de siglos anteriores, principalmente basado en la dinámica comercial y el abastecimiento de recursos, fue en el siglo XX cuando se consolidó a través de los sistemas de movilidad, el movimiento de la mano de obra - en ocasiones pendulares-, así como por las

Figura 2. Panorámica del tranvía del Oriente y la conexión Valle de Aburrá
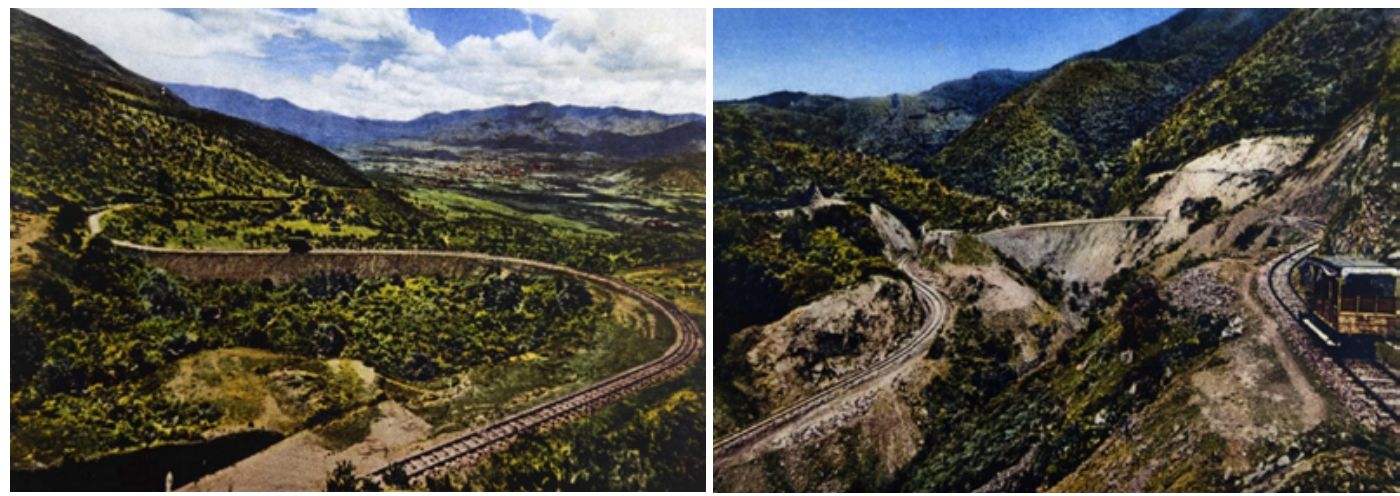

Fuente: archivo Biblioteca Pública Piloto. 
apuestas de articulación y ensanchamiento en los procesos de planificación de las dinámicas del Valle de Aburrá con el Valle de San Nicolás. De esta forma, el Oriente va a quedar inmerso en los procesos de expansión y concentración de Medellín, bajo la idea de ser el segundo piso o la zona de expansión en los proyectos políticos y económicos que se empiezan a implementar en este periodo, como se observa a continuación.

\section{El movimiento industrial al Oriente antioqueño}

Históricamente, el proceso de industrialización del departamento de Antioquia estuvo concentrado en su capital, la ciudad de Medellín, y en municipios como Envigado, Itagüí, Bello y Sabaneta (Ibiza, 1970), que orbitan a su alrededor. Pero desde los años 60, diversas unidades de producción y algunas fábricas completas se han trasladado a la subregión del Oriente antioqueño. Según el discurso empresarial y estatal que sustentó este movimiento, las motivaciones fueron el alto costo de la mano de obra en Medellín por la presencia de los sindicatos, pocas tierras disponibles, el incremento de la inseguridad, entre otros (Ibiza, 1970; Betancur et al., 2001). En contraste, el Oriente antioqueño ofrecía ventajas como:

1. Mano de obra relativamente calificada, con experiencia artesanal y menos costosa (el salario básico que se pagaba era menos de la mitad del que se pagaba en Medellín).

2. Terrenos a bajos precios y disponibles para la instalación de fábricas, en zonas rurales y alejados del crecimiento urbano.

3. Abundantes fuentes de agua, buen servicio eléctrico y sistema vial adecuado.

4. Incentivos fiscales como la exoneración al impuesto de industria y comercio, que podría oscilar entre los cinco y los 18 años.

5. Falta de planeación por parte de los municipios y un inadecuado control ambiental. (Ibiza, 1970; CORNARE e INER, 1993a).

Estos factores y las perspectivas exportadoras que se estaban abriendo con la internacionalización de la economía (Uribe, 1988, Villegas, 1988, CENIC, 1988 ) alentaron un proceso de "retorno" de actividades más tecnificadas a los municipios del Valle de San Nicolás, donde la procedencia de capital y la demanda de tierras obedecía a un proceso externo, es decir, no eran capitales locales que estuvieran regresando. Este "retorno" se enmarca en el interés de sectores políticos y económicos de ensanchar la subregión del Oriente a las dinámicas industriales, políticas y económicas asentadas en la capital. Esto configuró el "Proyecto Medellín”, al menos en los discursos e imaginarios de planeadores, políticos y actores sociales. En este periodo se afirmaba que el Oriente 
presentaba las mejores condiciones para la expansión de la capital y su industria, lo cual atrajo el interés de inversiones nacionales y extranjeras (CENIC, 1988).

Lo anterior fue estructurando al Valle de San Nicolas desde una funcionalidad ligada a la expansión del proyecto industrial, la cual se fue reforzando con la implantación de una infraestructura ligada a fortalecer los flujos extraterritoriales. Se destaca la creación de la autopista Medellín-Bogotá, inaugurada en 1980 y cuya construcción se empezó a pensar en 1959; el aeropuerto José María Córdoba, que inició su construcción en 1978 y finalizó en 1987; la Zona Franca de Rionegro, proyectada para su construcción a finales de los 80, inaugurada en 1993 y que inició operaciones en 1995; y el túnel de Oriente, que fue planeado en paralelo con la autopista en los años 60 y que inició operaciones en el año 2019. Vale la pena mencionar que recientemente se ha proyectado la expansión de la segunda pista del aeropuerto, que se espera inaugurar en 2023.

Este movimiento ha sido catalogado como un proceso de "desconcentración regionalmente concentrada" (Betancur et al., 2001) ya que la relocalización acontecida era principalmente de eslabones del proceso productivo, pero las sedes gerenciales continuaban asentadas en Medellín y/o el Valle de Aburrá. En los últimos años, la dinámica empieza a modificarse, existen empresas que, desde medianos del 2000 , se han relocalizado por completo, además de aumentar la llegada de nuevas funciones (administrativas, tecnológicas, de innovación) al Valle de San Nicolás. Sin embargo, la capital continúa siendo el epicentro tanto de personal calificado como de referencia global (C. Escobar, comunicación personal, 16 de noviembre de 2018).

Es de resaltar que la industria asentada no se esparció por el conjunto de municipios del Oriente antioqueño o del Valle de San Nicolás, como se ilustra en las figuras 3 a la 5 . Por el contrario, ha funcionado como un proceso de recorte del espacio rural de los municipios de Guarne, Rionegro, Marinilla y de algunas zonas del Carmen de Viboral, La Ceja y El Retiro, que están próximas a la infraestructura de movilidad creada, como se observa en la figura 3.

La figura anterior detalla la ubicación de las cerca de 240 industrias manufactureras (pequeñas, medianas y grandes) en el Valle de San Nicolás (puntos amarillos) y las zonas donde se concentró. Las figuras 4 y 5 permiten ubicar la concentración de empresas por año de ubicación y tamaño. Entre más empreas se encuentren próximas, mayores son los grados de densidad empresarial, como se ilustra en la figura 4, donde se describe la densidad por año de ubicación o la figura 5 , donde se detalla la densidad por el tamaño de la empresa asentadas, lo que permite interpretar cómo la agrupación fue forjando unos recortes muy marcados que se han extendido por la zona de Belén (Rionegro), la autopista 
Figura 3. Asentamiento industrial en el Valle de San Nicolás, Oriente antioqueño

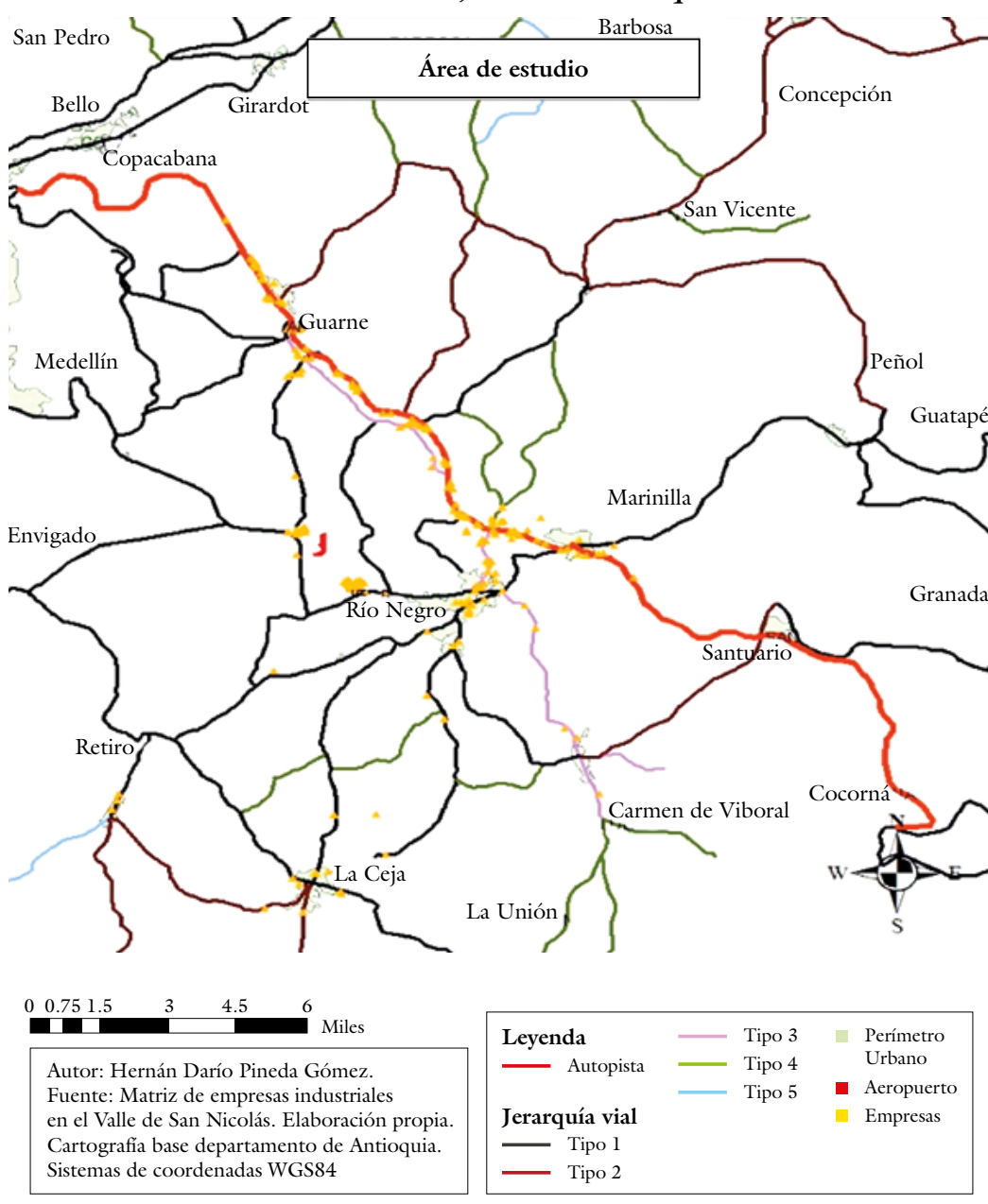

Fuente: elaboración propia

\section{territarias 45}

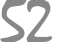

Medellín-Bogotá entre Guarne y Marinilla (línea negra en la figura) y las inmediaciones del aeropuerto José María Córdova. De esta forma, la industria ha configurado una serie de polígonos o recortes al interior del Valle de San Nicolás.
De acuerdo con diversos estudios (Ibiza, 1970; Franco, 2006; Salazar, 2010; Montoya, 2016), la llegada de la industria al Oriente ha sido el detonante de una serie de cambios como el tránsito de un paisaje rural a uno suburbano, la pérdida de la vocación tradicional campesina, el aumento poblacional, entre otros, configurándose como un proceso de regionalización que ha ido moldeando las dinámicas presentes. Además, la cercanía y articulación con el Valle de Aburrá y la dinámica de urbanización del Valle de San Nicolás ha aportado a la producción de sentidos de tiempo y nociones de progreso diferenciadas en la subregión del Oriente, fragmentándolo en dos grandes espacios geográficos: un oriente lejano, que hace alusión a aquellos municipios más apartados y con dinámica mucho más rural; y un oriente cercano (el Valle de San Nicolás), ligado a la dinámica de Medellín y con un ritmo más acelerado (Salazar, 2010; Aramburo \& García, 2011). Esto implica una ruptura con la idea de Oriente como espacio homogéneo.

\section{Acciones institucionales para impulsar la industria en el Oriente antioqueño}

El proceso geopolítico de configuración territorial desplegado a partir del asentamiento industrial ha sido dinamizado por diversos conglomerados económicos, con un rol protagónico de instancias del sector público, que han promovido la generación 
Figura 4. Densidad de empresas por año de ubicación en el Valle de San Nicolás

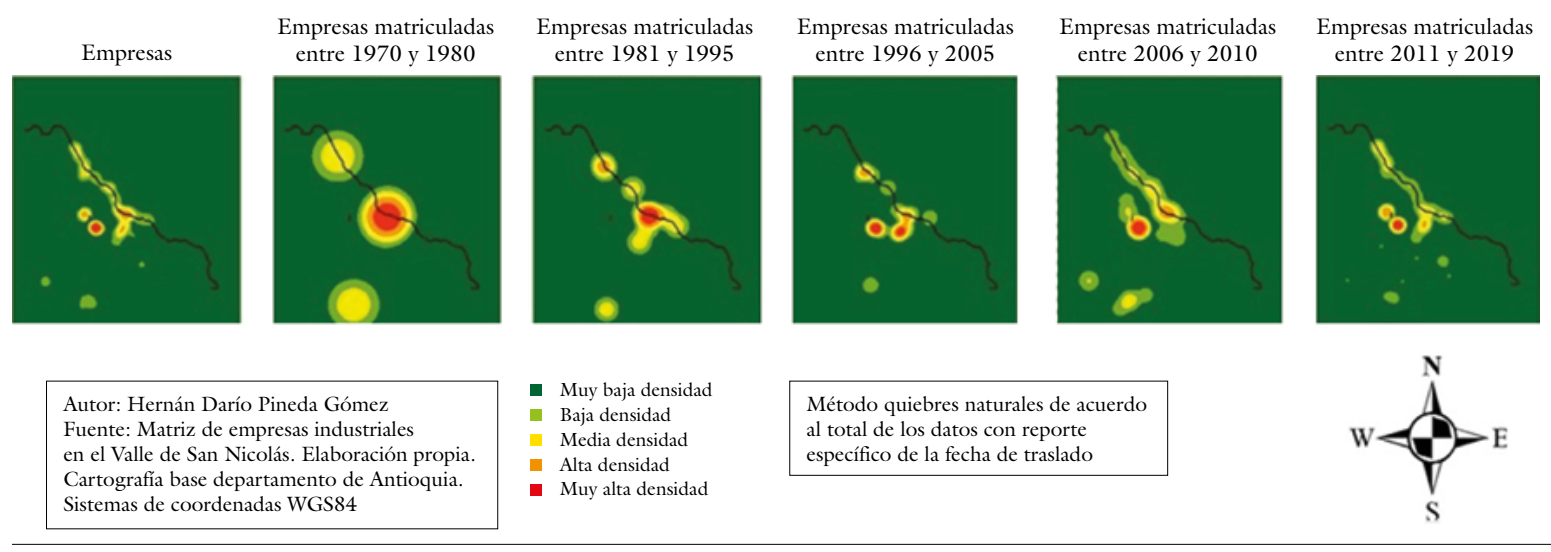

Fuente: elaboración propia.

Figura 5. Densidad de empresas por tamaño en el Valle de San Nicolás

Empresas
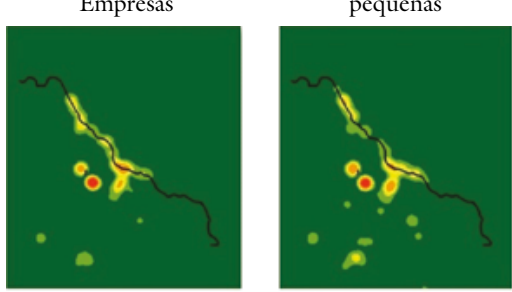

Autor: Hernán Darío Pineda Gómez

Fuente: Matriz de empresas industriales

en el Valle de San Nicolás. Elaboración propia.

Cartografía base departamento de Antioquia.

Sistemas de coordenadas WGS84

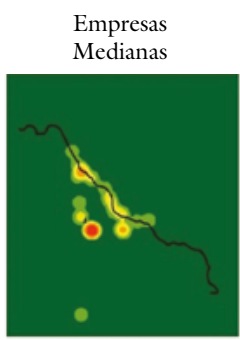

- Muy baja densidad

- Baja densidad

-1 Media densidad

- Alta densidad

- Muy alta densidad
Empresas

grandes
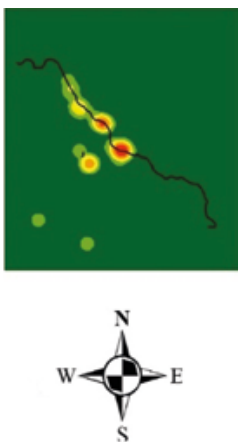

Método quiebres naturales de acuerdo

al total de los datos con reporte

específico de tamaño de la empresa

Fuente: elaboración propia.

de condiciones para la ubicación de estas actividades, bajo un imaginario de desarrollo regional articulado/dependiente con el Valle de Aburrá. Su direccionamiento ha partido de una serie de apuestas supramunicipales, así como la construcción de una institucionalidad público-privada. En algunos periodos, particularmente finales de los 70 y los años 80 , se complementaron con directrices nacionales orientadas a la desconcentración industrial, como el plan de desarrollo 1974-1978. Estas políticas recurrían a medidas como la creación de 
parques industriales y las restricciones a la inversión extranjera directa en los principales núcleos urbanos del país. Esto llevó a que empresas multinacionales COMO ARCLAD, TANN Y LABORATORIOS GRIFFIN se ubicaran en municipios como Rionegro, La Ceja y Marinilla.

La estructuración de una institucionalidad para planear y ordenar el espacio geográfico, en función del movimiento industrial, ha estado marcada por el traslado de competencias legales entre entidades públicas. En un primer momento, la planeación e intervención de la subregión era liderada desde la oficina de Planeación Departamental, a través de la Dirección de desarrollo del Oriente Cercano, aproximadamente hasta 1985. Posteriormente, este rol es asumido por CORNARE, entidad creada en 1983 por la Ley 60 del mismo año, con la función específica de la planeación del desarrollo regional. En 1993 le es removida esta función y pasa a ser autoridad ambiental (Ley 99 de 1993). En este período se crea la asociación de municipios del altiplano, MASORA 1992, entidad que pretendió coordinar acciones tendientes a propiciar un modelo de ocupación regional coordinado. El tránsito entre entidades públicas se ha materializado a través de empalmes de apuestas estratégicas como: el Plan de Ordenamiento Espacial (1983), El Oriente Antioqueño hacia el siglo XXI: construcción concertada del futuro (1990), Protocolo para el desarro-

\section{territarias 45} llo regional (1991), el Proyecto Pueblos
(1993-1996) y el Acuerdo para simultaneidad en el ordenamiento territorial (1997), este último como desarrollo de las competencias asignadas a los municipios para el ordenamiento del territorio en la Ley 388 de 1997. En este sentido, el proyecto normativo para intervenir y regir el Valle de San Nicolás se configura como un proceso extendido en el tiempo.

Estos ejercicios de planeación e intervención hacia el Oriente están cimentados en una visión de región competitiva, que tiene como núcleo central el Valle de Aburrá (Betancur et al., 2001; Franco, 2006). Esto implica que la interlocución y la planeación de la localización industrial se haya realizado en otras escalas del poder político y económico. La materialización de estas iniciativas no se puede asumir de forma lineal, pero su elaboración hace parte de estrategias de modernización selectiva, donde primero se genera la "necesidad" o proyecto futuro como condición para luego intervenir (Silveira, 2007). De esta forma, la competitividad se estructuró en las últimas décadas como la variable que dinamiza la organización territorial.

Esta institucionalidad y los planes trazados han funcionado como instrumento de intervención, como una forma de recortar el Valle de San Nicolás a partir de habilitar determinados usos del suelo en esta subregión, priorizando amplios fragmentos para actividades urbano/industriales y estructurando una imagen de región normativizada. Por ello, se puede 
afirmar que esta forma de proyectar la ocupación del Valle de San Nicolás ha operado desde unas territorialidades del orden y con la lógica de apropiar, delimitar y producir territorios a partir del estímulo de ciertos usos que están ligados a una idea de proyecto regional.

\section{Lógica territorial de la industria asentada}

Los recortes territoriales producidos por la proliferación de polígonos industriales, generalmente en las afueras de los perímetros urbanos municipales (figura 6), operan como enclaves en el proceso productivo. La materia prima que llega se transforma y se producen ciertas mercancías, luego se utiliza la infraestructura y conexión a mercados nacionales e internacionales para distribuirlas. Una característica de esta dinámica es que la forma de delimitación territorial opera a partir del movimiento desde y hacia el polígono industrial y del uso de la infraestructura instalada para articular los eslabones de la producción, la distribución y el gerenciamiento. Esto propicia un anclaje espacial para las actividades económicas, que como característica histórica presenta una desconexión con la dinámica local (Uribe, 1988; Villegas, 1988; Franco, 2006; Concejo Municipal de Guarne, 2016), lo que permite interpretarla como un fenómeno de ocupación que ha forjado zonas de gran concentración, particularmente en el triángulo que forman los municipios de Rionegro, Guarne y Marinilla.

Figura 6. Distancia de la industria a los municipios en el Valle de San Nicolás

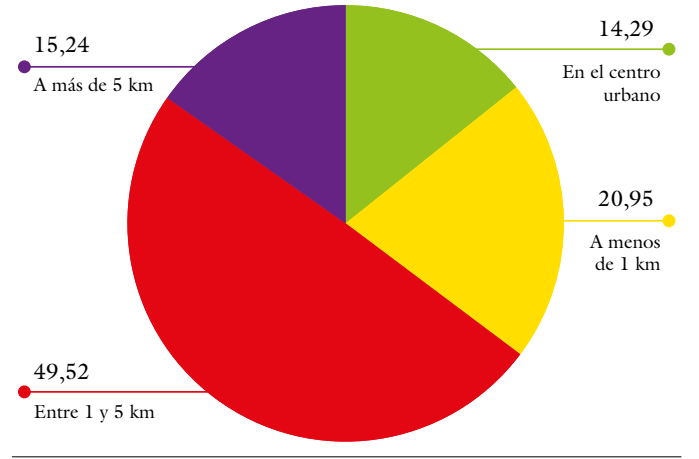

Fuente: elaboración propia a partir de la cartografía de industrias en el Valle de San Nicolás.

Este proceso ha sido dinámico y, de acuerdo con el análisis de los tiempos de asentamiento, las políticas en cada periodo y las demandas inmersas, se pueden agrupar en cinco grandes momentos:

1. Un asentamiento disperso de la industria en el suelo rural del Valle de San Nicolás entre 1960 y 1980. De acuerdo con las demandas empresariales en este periodo (Ibiza, 1970, Villegas, 1988, Salazar, 2010), su lógica obedece a una idea de territorialidades espontáneas, donde la necesidad de recursos, las posibilidades de expansión y las promesas de nuevas infraestructuras son el motor de este proceso. 
2. Un segundo periodo caracterizado por una anticipación espacial, producto de las proyecciones de grandes obras de infraestructura como la autopista Medellín-Bogotá y el aeropuerto internacional. Este periodo abarca desde mediados de los años 70 hasta inicios de los años 80 .

3. Un tercer momento caracterizado por una amplia densidad normativa. Esta fase está asociada a unas territorialidades del orden, donde la norma impulsa un determinado uso y propicia una selectividad de fragmentos del Oriente para el asentamiento industrial. Se extiende desde inicios de los años 80 hasta mediados de los años 90.

4. Un cuarto periodo de contención territorial asociado a una mayor intensidad del conflicto armado en esta subregión administrativa del departamento, aproximadamente de 1996 a 2006. Fueron pocas las empresas reubicadas y se podría caracterizar este proceso como de territorialidades contenidas.

5. Finalmente, un quinto momento que inicia aproximadamente en el 2004 y se extiende hasta la fecha. Se configura como un periodo de reagrupación espacial del proyecto competitivo a partir de nuevas y viejas iniciativas, en el cual aumentan las empresas relocalizadas y se retoman apuestas supramunicipales para la organización funcional de décadas anteriores. En este periodo, la lógica territorial en el polígono industrial opera a partir de unas territorialidades "corporativas fragmentadas" de borde.

Los anteriores elementos permiten interpretar que estos recortes producidos por la industria (figura 5) no están aislados, están inmersos en una lógica territorial que envuelve el Valle de San Nicolás. Este movimiento, con alcance a diferentes escalas, produce un territorio corporativo de borde de la metrópoli, concepto que se usa en este contexto para interpretar el funcionamiento de polígonos industriales, las dinámicas urbanizadas adyacentes a él y el vínculo con los lugares de dirección. Este proceso se estructura a partir de la comprensión de las formas (gran predio industrial, zona franca, bodega, parque industrial), los tipos de asentamiento industrial (empresa relocalizada, planta relocalizada, empresa nueva, ampliación de planta), el circuito espacial de producción (de dónde traen la materia prima y hacia dónde va la mercancía), el movimiento y procedencia de la mano de obra y la relación entre el centro gerencial/centro productivo (en referencia con el direccionamiento estratégico y el flujo de ordenes). En su conjunto representan una forma específica de regionalizar, de diferenciación geográfica, de dominio y control, es decir, una forma de producir territorios particulares.

El funcionamiento de los polígonos industriales está asociado a una forma de territorialización de la industria. Este 
proceso no acontece sobre el conjunto de la subregión administrativa, sino opera sobre fragmentos, dejando incluso intersticios en el proceso, huecos ocupados por otras dinámicas territoriales. A estas territorialidades no les interesan los límites existentes, ya sean administrativos o de apropiación local, se rigen por la búsqueda de una productividad espacial, por ello las espacialidades producidas obedecen a un hibrido entre prácticas, instituciones, relaciones de poder y anclajes espaciales para la rentabilidad del capital. De esta forma, se forjan unos espacios particulares de la globalización como resultado de la transformación de este espacio local, donde los polígonos industriales actúan formando una imagen de región construida desde los recortes que han producido.

\section{Territorialidades permeables en el Oriente antioqueño}

Hacia el año 1960, la zona del Valle de San Nicolás en el Oriente antioqueño se caracterizaba por ser una zona predominantemente agraria (Montoya \& Carmona, 2020), al menos hasta la década de los 70, periodo desde el cual se ha combinado la economía campesina de parcela con la agricultura comercial como el cultivo de flores (Molina, 1997). Su configuración en los años 60 consistía en pequeños núcleos urbanos que apenas se estaban construyendo y que dependían directamente de las dinámicas productivas
Figura 7. Apuesta de organización de actividades en el Valle de San Nicolás años 90

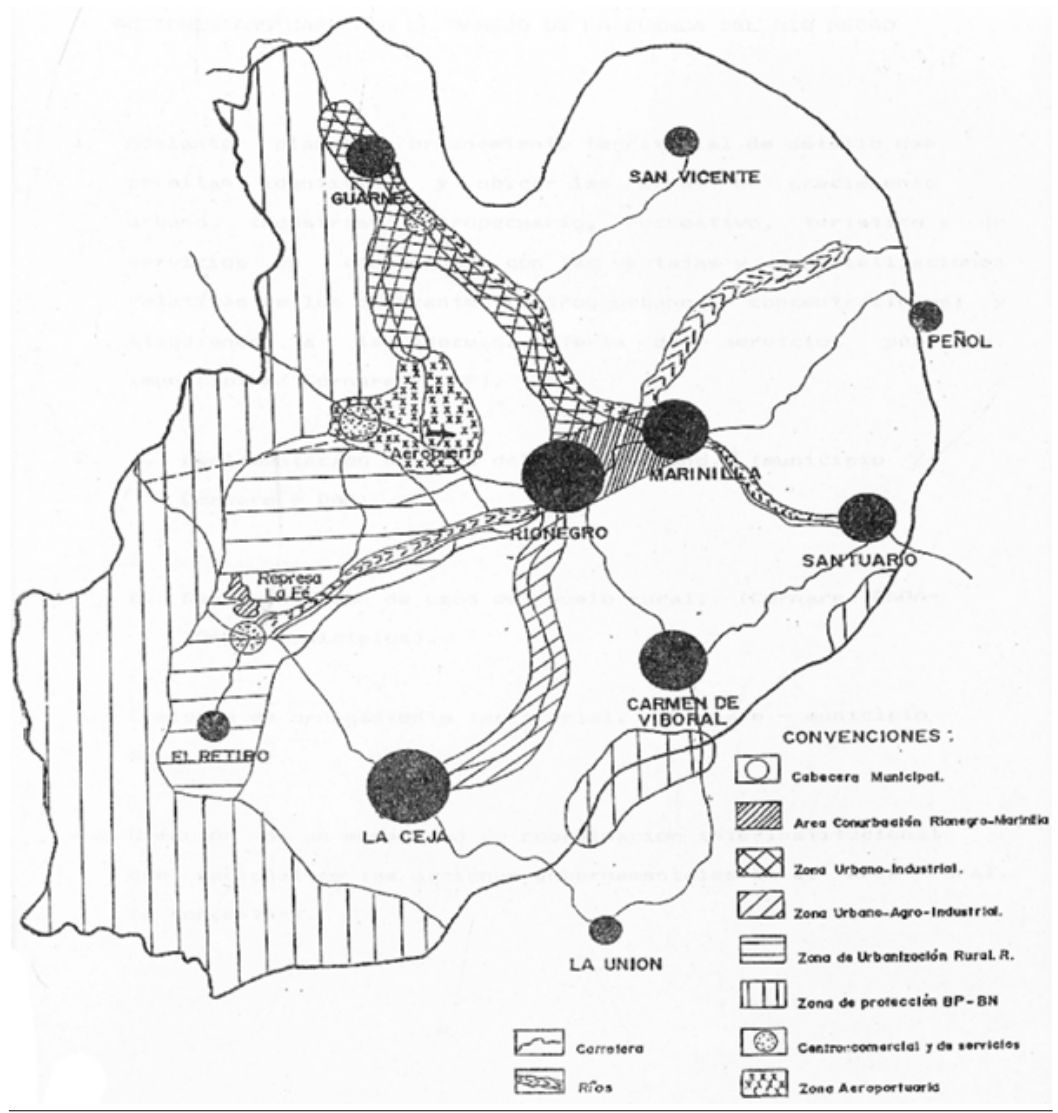

Fuente: CORNARE (1990).

rurales, los cuales se fueron transformando principalmente por la demanda de suelos urbano-industriales en los últimos 50 años (Salazar, 2010).

La llegada de una industria más tecnificada conllevó a una descomposición de la economía campesina y la migración de parte de esta población a áreas urbanas 
(Villegas, 1988; CENIC, 1988, Salazar, 2010), así como un deterioro de actividades artesanales ante la hegemonía de nuevos sistemas técnicos que establecieron otra forma de producir. Durante los años 80 e inicios de los 90, diversas apuestas de planeación plantearon la división del Valle de San Nicolás en dos grandes fragmentos (figura 7): el primero, en proceso de urbanización y bajo la influencia de la expansión de dinámicas con centralidad metropolitana, abarcaba a Rionegro, Guarne, La Ceja y El Retiro, la entrada a Marinilla y el Carmen de Viboral; el segundo, con proyección hacia un Distrito Agrario, principalmente el sector más oriental del Altiplano comprendido por El Santuario, San Vicente, El Carmen de Viboral, La Unión y Marinilla (CORNARE, 1990). No obstante, en la práctica, la disponibilidad de suelos urbanizables y los pocos controles no lograron la armonización planteada (Montoya \& Carmona, 2020) y la producción campesina fue desplazada a zonas periféricas de los municipios, con posibilidades cada vez menores de producción rentable.

La dinámica campesina y la industria artesanal se fueron moldeando por los ritmos, tiempos y formas de articulación territorial, trazados por los proyectos de organización funcional para esta subregión. La hegemonía de actividades urbano-industriales en los corredores de movilidad, principalmente en la zona rural de los municipios objeto de aná- fragmentado en el Valle de San Nicolas. Estas actividades han consistido principalmente en el establecimiento de grandes predios industriales, parques industriales, espacios para el almacenamiento de mercancías, grandes infraestructuras como la Zona Franca o el aeropuerto José María Córdova, así como la dispersión de vivienda tipo parcelación y/o condominio, y una explosión de actividades comerciales.

Como efecto de la fragmentación producida por las actividades urbanoindustriales, la dinámica territorial del Oriente se expresa en una triple lógica: una ligada a esa idea de Oriente desde la periferia campesina de subsistencia en las zonas más apartadas de los ejes de fluidez territorial; una segunda, desde una idea más urbanizada, ligada a una visión de Oriente desde los flujos y ritos de la urbanización; y la tercera, intersticial, asociada a los espacios no ocupados por los polígonos industriales, una territorialidad que se acerca más a una idea de aglomerado, en los términos planteados por Haesbaert (2019).

Aún en este contexto, persiste un proyecto político-social de Oriente que busca cobijar al conjunto de los 23 municipios, configurado como una idea de proyecto autónomo y en respuesta a los efectos negativos del desarrollo regional impuesto. Esta forma de concebir el Oriente tanto en el discurso como en la práctica implica un proyecto de región y de identidad. lisis (figura 3 ), ha producido un paisaje Sin embargo, este proyecto de Oriente es permeado por los proyectos económicos,

\section{territarias 45}


"condenado" de forma inevitable a las maneras en cómo se van construyendo los territorios en un mundo globalizado y urbanizado.

\section{A modo de conclusión: la región segmentada-superpuesta y la producción de retazos espaciales}

Los elementos expuestos en los anteriores literales ilustran cómo la localización industrial en el Valle de San Nicolás ha estado enmarcada por una serie de planes, discursos y tensiones entre actores y prácticas, produciendo tanto tiempos e imaginarios del desarrollo como unas espacialidades propias del proceso. Las empresas que se han asentado han pretendido darle un uso particular y privatizado al territorio, centrado en las relaciones entre la unidad de producción, la unidad gerencial y la proyección de salto a lo global. De esta forma, estos recortes se configuran como un espacio particular de la globalización.

El fragmento industrial y sus alrededores se han pretendido usar como plataforma de inserción global. Son las verticalidades las que intentan definir, desde lugares externos, la funcionalidad de cada fragmento y su conexión con flujos a otras escalas. Las territorialidades en torno a la industria han fragmentado el espacio rural del Oriente Cercano, configurando unos polígonos de borde que no se entrelazan a las dinámicas locales, pero las afectan. Medellín se ha estructurado como el centro de gravedad de esta fragmentación territorial. En este sentido, las diversas formas de envolver el Oriente han producido recortes territoriales como una forma superpuesta de producir región. Entre ellas encontramos:

- Un proyecto de región normativizada: una apuesta por la intervención supramunicipal y local.

- La región tecnifica como agujerosred, una idea de macro-región funcional. Se configura una idea de Oriente articulada al Valle de Aburrá desde los agujeros o recortes territoriales (polígonos industriales), una forma centralizada/dependiente.

- La Región-zona, un lugar agujereado, una idea de región más horizontal desde el proyecto político social, fragmentada por el proyecto económico.

- Una idea de regionalización desde los intersticios, una forma de ver las dinámicas en los agujeros no ocupados por la industria.

Estos elementos permiten centrar la discusión en la producción superpuesta de un proyecto de región estructurado en/a partir de la volatibilidad del "Proyecto Medellín", que, si bien nominalmente se acoge en el mismo nombre, representa relaciones de poder, significaciones y prácticas distintas. Esta producción funcional del territorio se complementa con una 
producción normativa que ha propiciado un uso funcional, un tipo de recorte, y se tensiona con el proyecto político social, configurándose una multiplicidad de formas de recorte territorial, donde las dinámicas territoriales horizontales se vuelven permeables ante las acciones del proyecto competitivo.

\section{Referencias}

IDOM. (2018). Modulo 3: Estudio de huella urbana y escenarios de crecimiento para el municipio de Rionegro (Antioquia -Colombia). En Estudio de buella urbana y escenarios de crecimiento para el municipio de Rionegro (Antioquia-Colombia). IDOM; Alcaldía de Rionegro; Banco Interamericano de Desarrollo, BID; FINDETER.

Aramburo, C. I., \& García, C. I. (Eds.). (2011). Geografias de la guerra, el poder y la resistencia. Oriente Antioqueño y Urabá. 1998-2008. Códice Ltda.

Betancur, M. S., Stienen, A., \& Urán, O. A. (2001). Globalización: cadenas productivas y redes de acción colectiva: reconfiguración territorial y nuevas formas de pobreza y riqueza en Medellín y el Valle de Aburrá. Tercer Mundo.

Fundación Codesarrollo. (1976). Oriente Cercano, un Polo de Desarrollo. Autoedición.

Concejo Municipal de Guarne. (2016). Plan de Desarrollo Municipal de Guarne. colectiva de Futuro, 2016-2019" [Acuerdo 004]. Alcaldía de Guarne.

Corporación Autónoma Regional de las Cuencas de los Ríos Negro y Nare, COrnare. (1990). El Oriente Antioqueño hacia el Siglo XXI. Autoedición. Corporación Autónoma Regional de las Cuencas de los Ríos Negro y Nare, CORNARE e Instituto de Estudios Regionales, INER. (1993a). Guarne, Antioquia. Colección de estudios de localidades. Universidad de Antioquia. Corporación Autónoma Regional de las Cuencas de los Ríos Negro y Nare, CORNARE e Instituto de Estudios Regionales, INER. (1993b). La Ceja, Antioquia. Colección de estudios de localidades. Universidad de Antioquia. Corporación Autónoma Regional de las Cuencas de los Ríos Negro y Nare, CORnARE. (2016). Actividad económica actual en el Oriente Antioqueño y perspectivas de crecimiento verde y desarrollo compatible con el clima. En Plan de Crecimiento Verde y Desarrollo Compatible con el Clima para el Oriente Antioqueño (anexo 4). Cornare, CDKN; Fundación Natura; WWF.

Corporación para las Iniciativas de Red de Educación en California, CENIC. (1988). Determinantes sociales y culturales de la planeación en la región Rionegro-Nare. Fase I Municipios: Puerto Triunfo, Sonsón, Marinilla, El Peñol, Rionegro y San Vicente. 19871988. Universidad de Antioquia 
Franco, V. (2006, junio). Centros de poder económico y político en Medellín: dilemas estructurales y selectividad estratégica. Controversia, 186, 90110. http://biblioteca.clacso.edu.ar/ Colombia/cinep/20100925011331/ conflictoyreligioncentrosdepoderControversial86.pdf

Haesbaert, R. (2010, enero-junio). Región, regionalización y regionalidad: cuestiones contemporáneas. Antares, 3, 2-23.

Haesbaert, R. (2014). Lógica zonal y ordenamiento territorial: Para rediscutir la proximidad y la contigüidad espaciales. Cultura y representaciones sociales, 8(16), 9-29.

Haesbaert, R. (2019). Regional-global. Dilemas de la región y de la regionalización en la geografía contemporánea. CLACSO; Facultad de Filosofía y Letras, UBA; Universidad Pedagógica Nacional.

Ibiza, G. (1970). Un ensayo sobre la descentralización industrial -el caso Medellín. [Documento de trabajo No. 4]. Centro de Investigaciones Económicas, Facultad de Ciencias Económicas de la Universidad de Antioquia.

Montoya, E. (2016). Agentes del capital en el discurso de la planeación en el Altiplano del Oriente Antioqueño. En C. Arango (Ed), Desarrollo y territorio: Perspectivas, abordajes, experiencias (pp. 47-64). Centro de Publicaciones Universidad Católica de Oriente.
Montoya, E., \& Carmona, L. S. (2020). La planeación del oriente antioqueño: el camino hacia la gran región metropolitana. En A. González, E. Montoya, E. Tôrres, M. Zerbone, L. S. Carmona (Comp.), Lectura territorial del oriente cercano antioqueño (pp. 17-36). Universidad Pontificia Bolivariana. http://doi.org/10.18566/978958-764-861-4

Molina, G. (1997). La Ciudad-Región: el área metropolitana del Valle de Aburrá y su relación con el Oriente cercano. Editorial Etcétera.

Salazar, S. (2010). Agentes industriales y cultivo de flores en la expansión Metropolitana de Medellín, Colombia. IV Jornadas de geografía económica. León, España.

Santos, M. (1993). Los espacios de la globalización. Anales de Geografía, 13, 69-77.

Santos, M., \& Silveira, M. L. (2008). O Brasil: território e sociedade no inicio do século XXI. Record.

Sassen, S. (2007). El reposicionamiento de las ciudades y regiones urbanas en una economía global: ampliando las opciones de políticas y gobernanza. EURE, 33(100), 9-34. http:// dx.doi.org/10.4067/S025071612007000300002

Silveira, M. L. (2007). Los territorios corporativos de la globalización. Geograficando, 3(3), 13-26. http://sedici. unlp.edu.ar/handle/10915/13941 
Silveira, M. L. (2008, septiembre-diciembre). Globalización y territorio usado: imperativos y solidaridades. Cuadernos Cendes, 25(69), 1-19.

Silveira, M. L. (2014). El territorio usado, un caleidoscopio de divisiones del trabajo. GEO SUR, 5(7), 15-34. http:// www.revgeosur.udec.cl/wp-content/ uploads/2016/08/Vol5N7_silveira. pdf
Uribe, J. G. (1988). La industrialización en el Oriente Antioqueño. En Determinantes sociales y culturales de la planeación de la región Rio Negro-Nare (anexo 3). Centro de Investigaciones Sociales, Universidad de Antioquia.

Villegas, L.C. (1988). La industria manufacturera en el oriente antioqueño. Centro de Investigaciones Sociales, Universidad de Antioquia; CORNARE. 